\title{
Structural basis of AAUAAA polyadenylation signal recognition by the human CPSF complex
}

\author{
Marcello Clerici $^{1}$, Marco Faini ${ }^{2}$, Lena M. Muckenfuss ${ }^{1}$, Ruedi Aebersold ${ }^{2,3}$, Martin Jinek ${ }^{1, *}$ \\ ${ }^{1}$ Department of Biochemistry, University of Zurich, Winterthurerstrasse 190, CH-8057 Zurich, \\ Switzerland ${ }^{2}$ Department of Biology, Institute of Molecular Systems Biology, ETH Zurich, \\ $\mathrm{CH}-8093$, Zurich, Switzerland ${ }^{3}$ Faculty of Science, University of Zurich, Switzerland
}

\begin{abstract}
Mammalian mRNA biogenesis is dependent on specific recognition of a hexanucleotide AAUAAA motif in the polyadenylation signals (PAS) of pre-mRNA transcripts by the cleavage and polyadenylation specificity factor (CPSF) complex. Here we report a $3.1 \AA$ resolution cryoelectron microscopy structure of a core CPSF module bound to the PAS hexamer motif. The structure reveals molecular interactions responsible for base-specific recognition, providing a rationale for mechanistic differences between mammalian and yeast 3' polyadenylation.
\end{abstract}

In mammalian cells, a central hexamer $\mathrm{A}(\mathrm{A} / \mathrm{U}) \mathrm{UAAA}$ motif is a key cis-acting element within the polyadenylation signal (PAS) required for 3' cleavage and polyadenylation of premRNA transcripts ${ }^{1-3}$. The PAS hexamer motif is specifically recognized by the Cleavage and Polyadenylation Specificity Factor (CPSF), a multi-subunit complex responsible for polyadenylation site definition, pre-mRNA cleavage and polyA-polymerase recruitment, and for coupling 3' end formation with transcriptional termination ${ }^{4-9}$. A core CPSF assembly composed of proteins CPSF160, WDR33, CPSF30 and Fip1 binds the A(A/U)UAAA motif, via direct interactions with CPSF30 and WDR33, and is sufficient to recruit polyApolymerase in vitro ${ }^{10,11}$. Despite the critical function of CPSF in mammalian mRNA biogenesis, the molecular interactions that underpin its specificity for the PAS hexamer motif are poorly understood.

To address this question, we reconstituted by coexpression in baculovirus-infected insect cells a core human CPSF polyadenylation module consisting of full-length CPSF160, an Nterminal fragment of WDR33 containing the WD40 domain, a truncated CPSF30 fragment

Correspondence should be addressed to M.J. (jinek@bioc.uzh.ch).

Data availability

The cryo-EM density map has been deposited in the Electron Microscopy Data Bank under accession code EMD-4225. The corresponding atomic model has been deposited in the Protein Data Bank under accession code 6FBS.

Author Contributions

M.C. and M.J. designed experiments. M.C. prepared all samples, and determined the cryo-EM structure of the CPSF160-WDR33CPSF30-PAS RNA complex. L.M.M. assisted with cryo-EM sample preparation. M.F. assisted with cryo-EM data analysis and data interpretation, supervised by R.A. M.C., M.F. and M.J. wrote the manuscript.

Competing Financial Interests

The authors declare no competing financial interests. 
containing zinc-finger (ZF) domains ZF1-ZF5 and a conserved fragment of Fip1 corresponding to residues Gly130-Lys195 ${ }^{\mathrm{Fip} 1}$, bound to a 10-nucleotide RNA containing the AAUAAA hexamer (Supplementary Fig. 1a). Using cryo-electron microscopy (cryo-EM) and single-particle analysis, we determined the structure of the core CPSF-AAUAAA complex at an overall resolution of $3.1 \AA$, as indicated by the Fourier shell correlation (FSC) plot (Supplementary Fig. 1b-g). The structure comprises full-length CPSF160 (residues Met1-Phe1443 ${ }^{\text {CPSF160}), ~ W D R 33 ~(L e u 41-L y s 410 W D R 33), ~ d o m a i n s ~ Z F 1-3 ~ o f ~ C P S F 30 ~(M e t 1-~}$ Ile $115^{\mathrm{CPSF} 30}$ ) and an RNA fragment containing the AAUAAA hexamer and two additional 3'-terminal nucleotides (Fig. 1a). No density could be observed for Fip1 and CPSF30 domains ZF4 and ZF5, suggesting that these domains form a flexible module. This is in good agreement with previous interaction studies showing that yeast Fip1 is an intrinsically disordered protein that interacts with Yth1 (the yeast CPSF30 ortholog) via its ZF4 and ZF5 domains ${ }^{12}$.

Overall, the structure of the human CPSF160-WDR33-CPSF30 complex is highly similar to a recently determined structure of the core polyadenylation module of the yeast CPF complex comprising Cft1 (the yeast CPSF160 ortholog), Pfs2 (WDR33) and Yth1 $(\text { CPSF30 })^{13}$ (Fig. 1b, Supplementary Figure 2a). The tandem ZF1-3 domains of CPSF30 line a cleft at the CPSF160-WDR33 interface near the N-terminal extension of WDR33 (Fig. 1c). The N-terminal region of CPSF30 upstream of the ZF1 domain extends over the surface of the CPSF160 beta-propeller domain bP3, inserting its N-terminus into the central cavity of bP3. These findings are in good agreement with recent biochemical characterization of subunit interactions within the human CPSF core module and an X-ray crystallographic structure of the CPSF160-WDR33 heterodimer ${ }^{14}$.

The ribose-phosphate backbone of the AAUAAA hexamer adopts an S-shaped conformation (Fig. 2a, Supplementary Figure 2b). The RNA is clamped at the CPSF30-WDR33 interface, establishing an intricate network of interactions that involve both the RNA backbone and the nucleobases (Supplementary Figure 3). The N-terminal region of WDR33 comprising residues Leu41-Lys55 ${ }^{\mathrm{WDR} 33}$, folds over the RNA, contacting nucleotides U3, A5 and A6 and thereby locking the RNA in place (Fig. 2a). The basic residues Lys46WDR33, $\operatorname{Arg} 47^{\text {WDR } 33}, \operatorname{Arg} 49^{\text {WDR } 33}$ and Arg54 ${ }^{\text {WDR } 33}$ contact the ribose-phosphate backbone of the RNA (Supplementary Figure 3). The centerpiece of the PAS hexamer is a Hoogsteen base pair formed between nucleotides U3 and A6 (Fig. 2b), sandwiched by $\pi-\pi$ stacking interactions with Phe $153^{\mathrm{WDR} 33}$ and Phe $43^{\mathrm{WDR} 33}$, both of which are invariant in mammalian WDR33 orthologs. Nucleotides A1-A2 are bound by the CPSF30 ZF2 domain, while nucleotides A4-A5 are bound by ZF3. The two segments of the AAUAAA hexamer are bound by the ZF domains in a nearly identical manner (Supplementary Fig. 2c). Altogether, these structural insights rationalize previous findings that implicated CPSF30 ZF2-3 domains and WDR33 N-terminus in specific recognition of the AAUAAA motif ${ }^{10,11,14}$

Each of the A1, A2, A4 and A5 bases occupies a pocket in CPSF30 in which the base stacks with a strictly conserved aromatic residue: A1 with Phe $84^{\mathrm{CPSF} 30}$, A2 with His70 ${ }^{\mathrm{CPSF} 30}$, A4 with Phe $112^{\mathrm{CPSF} 30}$, and A5 with Phe $98^{\mathrm{CPSF} 30}$ (Fig. 2c). The nucleobases of A1, A4 and A5 are further recognized via their Watson-Crick edges by sequence-specific hydrogen bonding interactions with their N1 and 6-amino groups (Fig. 2c). A1 is contacted by the main-chain 
amide of Lys69 ${ }^{\mathrm{CPSF} 30}$ and carbonyl of Val67 ${ }^{\mathrm{CPSF} 30}$; A4 by the main-chain amide of Tyr97 ${ }^{\text {CPSF30 }}$ and carbonyl of Glu95 ${ }^{\mathrm{CPSF} 30}$; A5 is contacted by the main-chain amide of Ser106 ${ }^{\mathrm{CPSF} 30}$ and side-chain carbonyl of Asn107 ${ }^{\mathrm{CPSF} 30}$. By contrast, the interaction with the nucleobase of A2 is less specific since a single hydrogen bond is established between the main-chain amide of Lys77 ${ }^{\mathrm{CPSF} 30}$ and the N1 group of the adenine base. Finally, the U3-A6 base pair does not appear to be recognized by base-specific interactions (Fig. 2b,c).

AAUAAA and AUUAAA are the two most common hexamer motifs found within the polyadenylation signals of mammalian mRNAs ${ }^{15-17}$. The observed base-specific recognition of adenosines at positions 1, 4 and 5 within the hexamer is consistent with their invariance in the consensus sequence. Conversely, the lack of base-specific recognition at position 2 is reflected by transcriptome-wide studies of mammalian mRNA polyadenylation showing that the second position within the PAS hexamer is the most variable ${ }^{15-17}$. Notably, the structural insights are also in good agreement with the observation that although the identity of each base within the PAS hexamer consensus strongly affects the efficiency of RNA cleavage and polyadenylation in a HeLa cell nuclear extract, the second position within the hexamer is the most permissive to base substitutions ${ }^{18}$. Hoogsteen base-pairing between nucleotides U3 and A6 explains their invariance in the PAS hexamer motif even in the absence of base-specific interactions with WDR33; although other isosteric pyrimidine-purine combinations could potentially fit in the U3-A6 binding pocket in WDR33, these base-pair combinations are energetically unfavorable due to non-optimal hydrogen bonding interactions, which likely results in substantially reduced affinity.

Whereas the AAUAAA hexamer is strongly conserved in mammalian pre-mRNA transcripts, the polyadenylation signals of yeast pre-mRNAs are less well-defined and typically contain degenerate A-rich motifs termed positioning elements ${ }^{19}$. Both structural superposition and sequence alignment of human CPSF30 and yeast Yth1 indicate that the A1, A4 and A5 recognition pockets are conserved in the Yth1 ZF2 and ZF3 domains (Supplementary Fig. 4a,b), implying that Yth1 recognizes adenosine nucleotides in positioning elements. In contrast to higher eukaryotic WDR33 orthologs, yeast Pfs2 lacks an aromatic residue equivalent to Phe 43 WDR33 in its N-terminal region (Supplementary Fig. 4c). As a result, the U3-A6 binding pocket is not conserved in Pfs2, possibly explaining why yeast positioning elements are degenerate and do not conform to a well-defined consensus sequence.

In conclusion, structural insights presented in this study reveal the molecular interaction framework that underpins polyadenylation site definition in mammalian pre-mRNA 3' end processing. While this manuscript was in preparation, another cryo-EM structure of the human CPSF160-WDR33-CPSF30 complex bound to the AAUAAA PAS motif was reported ${ }^{20}$, with similar conclusions. Despite emerging commonalities in the molecular architectures of yeast and mammalian cleavage and polyadenylation complexes, their structural features responsible for RNA binding have diverged to recognize distinct polyadenylation signals. Ongoing studies of yeast CPF and mammalian CPSF complexes will shed light on the molecular mechanisms that couple polyadenylation signal recognition to the nuclease and polyA polymerase enzymatic activities, as well as to transcriptional termination, and provide insights into their regulation. 


\section{Online Methods}

\section{Protein expression and purification}

Human CPSF subunit constructs were cloned into MacroLab vectors developed by Scott Gradia (University of California, Berkeley) ${ }^{21}$. His6-tagged full-length CPSF160 (Uniprot Q10570) was inserted into the 438B vector (Addgene \#55219); His $_{6}$-(StrepII) ${ }_{2}$-WDR33 (Uniprot Q9C0J8-1, residues 1-410) was inserted into a modified version of the 438B vector; untagged CPSF30 (Uniprot O95639-3, residues 1-178), was inserted into the 438A vector (Addgene \#55218); StrepII-GFP-tagged Fip1 (Uniprot Q6UN15-4, residues 130-195) was inserted into the 438Rgfp vector (Addgene \#55211) by ligation-independent cloning. The four constructs were combined in a single plasmid using the MacroBac ligationindependent cloning system ${ }^{21}$. Recombinant baculovirus was generated using the Bac-toBac system (Invitrogen) according to standard protocols and used to infect Sf9 cells at a density of $1.0 \times 10^{6} \mathrm{ml}^{-1}$. Cells were harvested $72 \mathrm{hr}$ post infection, resuspended in $20 \mathrm{mM}$ Tris-Cl pH 7.5, $200 \mathrm{mM} \mathrm{NaCl}, 10 \%$ glycerol and $0.05 \%$ Tween20, supplemented with Protease Inhibitor Cocktail (GE Healthcare), and lysed by sonication. The complex was purified on Ni-NTA resin (Qiagen), followed by purification on Streptactin superflow resin (IBA). Expression tags were removed by overnight incubation with TEV protease. Synthetic 10-mer PAS RNA (5'-ACAAUAAAGG-3') was added in 1.2-fold molar excess and the resulting protein-RNA complex was further purified by size exclusion chromatography on a Superdex-200 column (GE Healthcare) in $20 \mathrm{mM}$ HEPES pH 7.5, $140 \mathrm{mM} \mathrm{KCl}$ and $1 \mathrm{mM}$ DTT. Peak fractions (as indicated in Supplementary figure 1A) were collected and flashfrozen in liquid nitrogen.

\section{Cryo-EM sample preparationand data collection}

The CPSF-PAS RNA complex was vitrified on Quantifoil $\mathrm{Cu} 400$ mesh R1.2/1.3 grids previously glow-discharged for 15 seconds on each side. $4 \mu \mathrm{l}$ of sample at $0.3 \mathrm{mg} / \mathrm{ml}$ was deposited on the EM grid, incubated for 15 seconds, blotted for 7 seconds and plunge-frozen in liquid ethane-propane mixture using a FEI Vitrobot Mark IV at $22{ }^{\circ} \mathrm{C}$ and $100 \%$ humidity. Data collection was performed on a FEI Titan Krios microscope operated at $300 \mathrm{kV}$ and equipped with a Gatan K2 Summit detector and a Gatan Quantum GIF LS energy filter. Image acquisition and initial processing was managed by Focus ${ }^{22}$. Micrographs with a pixel size of $1.058 \AA$ were collected in super-resolution mode and consisted of 50 frames for a total exposure time of 10 seconds and a total dose of $80 \mathrm{e}^{-} / \AA^{2}$. The defocus ranged between -2.2 and $-1 \mu \mathrm{m}$.

\section{Image processing}

Motion correction and averaging were performed with MotionCor $2^{23}$. Final micrographs are dose-weighted sums of the full stack removing the first frame. Contrast transfer function (CTF) parameters were determined with CTFFIND4 ${ }^{24}$. Subsequent processing was performed in RELION-2 ${ }^{25}$. A dataset of 1070 images was selected with a CTF resolution estimate between 2.6 and $3.0 \AA$ A. 1600 particles were manually picked from a random subset of micrographs and the resulting 2D class averages used as input for the RELION-2 Autopick function on binned micrographs, obtaining a total 265,000 particles. After selection of 263,000 particles with best Autopick figure of merit, the particles were 
subjected to two rounds of 2D classification, selecting particles from 72 and 27 classes, respectively. The final dataset contained 137,000 particles representing $51 \%$ of the picked particle dataset. Aligned particles were then extracted from unbinned micrographs and an initial reference-free model was generated using RELION-2 (Initial model function). 3D classification of the dataset into 3 classes yielded models with similar features, number of particles and final resolution, suggesting structural homogeneity. Therefore, the whole dataset was directly subjected to 3D refine alignment. After post-processing in RELION-2 the final map reached a resolution of $3.1 \AA$. The resolution was measured by Fourier shell correlation between reconstructions from two independently refined half-datasets (goldstandard FSC in RELION-2). Local resolution was calculated within RELION-2 with ResMap ${ }^{26}$ from two data-independent reconstructions.

\section{Model building}

Model building was performed in $\operatorname{Coot}^{27}$ using the CPSF160-WDR33 heterodimer as starting model (PDB 6F9N) and refined using phenix.real_space_refine ${ }^{28}$. Figures were prepared with Chimera ${ }^{29}$ and PyMol (https://www.pymol.org)

\section{Supplementary Material}

Refer to Web version on PubMed Central for supplementary material.

\section{Acknowledgements}

We are indebted to H. Stahlberg for access to the Bio EM Lab at the C-CINA Center for Cellular Imaging and Nanoanalytics at the University of Basel. We thank L. Kovacik and M. Chani for technical assistance with cryo-EM image acquisition. We are grateful to J. Novacek and M. Peterek (Core Facility for Cryo-electron Microscopy and Tomography, CEITEC, Masaryk University, Brno, Czech Republic) for initial sample analysis. We are grateful to A. Scaiola at the ScopeM Scientific Center for Optical and Electron Microscopy at ETH Zurich for assistance with sample preparation and preliminary sample analysis. This work was supported by the European Research Council (ERC) Starting Grant ANTIVIRNA (Grant no. ERC-StG-337284). M.F. was supported by a Long-Term Fellowship from the European Molecular Biology Organization (EMBO ALTF-343-2013). R.A. acknowledges support from the European Union 7th Framework Program (PROSPECTS, HEALTH-F4-2008-201648), the European Research Council (ERC Advanced Grants no. 233226 and no. 670821), and the Innovative Medicines Initiative Joint Undertaking (ULTRA-DD, grant no. 115766). M.J. is International Research Scholar of the Howard Hughes Medical Institute and Vallee Scholar of the Bert L \& N Kuggie Vallee Foundation.

\section{References}

1. Proudfoot NJ, Brownlee GG. 3' non-coding region sequences in eukaryotic messenger RNA. Nature. 1976; 263:211-4. [PubMed: 822353]

2. Shi Y, Manley JL. The end of the message: multiple protein-RNA interactions define the mRNA polyadenylation site. Genes Dev. 2015; 29:889-97. [PubMed: 25934501]

3. Tian B, Graber JH. Signals for pre-mRNA cleavage and polyadenylation. Wiley Interdiscip Rev RNA. 2012; 3:385-96. [PubMed: 22012871]

4. Bienroth S, Wahle E, Suter-Crazzolara C, Keller W. Purification of the cleavage and polyadenylation factor involved in the 3'-processing of messenger RNA precursors. J Biol Chem. 1991; 266:1976876. [PubMed: 1918081]

5. Chan S, Choi EA, Shi Y. Pre-mRNA 3'-end processing complex assembly and function. Wiley Interdiscip Rev RNA. 2011; 2:321-35. [PubMed: 21957020]

6. Keller W, Bienroth S, Lang KM, Christofori G. Cleavage and polyadenylation factor CPF specifically interacts with the pre-mRNA 3' processing signal AAUAAA. EMBO J. 1991; 10:42419. [PubMed: 1756731] 
7. Murthy KG, Manley JL. Characterization of the multisubunit cleavage-polyadenylation specificity factor from calf thymus. J Biol Chem. 1992; 267:14804-11. [PubMed: 1634525]

8. Shi Y, et al. Molecular architecture of the human pre-mRNA 3' processing complex. Mol Cell. 2009; 33:365-76. [PubMed: 19217410]

9. Xiang K, Tong L, Manley JL. Delineating the structural blueprint of the pre-mRNA 3'-end processing machinery. Mol Cell Biol. 2014; 34:1894-910. [PubMed: 24591651]

10. Chan SL, et al. CPSF30 and Wdr33 directly bind to AAUAAA in mammalian mRNA 3' processing. Genes Dev. 2014; 28:2370-80. [PubMed: 25301780]

11. Schonemann L, et al. Reconstitution of CPSF active in polyadenylation: recognition of the polyadenylation signal by WDR33. Genes Dev. 2014; 28:2381-93. [PubMed: 25301781]

12. Barabino SM, Ohnacker M, Keller W. Distinct roles of two Yth1p domains in 3'-end cleavage and polyadenylation of yeast pre-mRNAs. EMBO J. 2000; 19:3778-87. [PubMed: 10899131]

13. Casanal A, et al. Architecture of eukaryotic mRNA 3'-end processing machinery. Science. 2017; 358:1056-1059. [PubMed: 29074584]

14. Clerici M, Faini M, Muckenfuss LM, Aebersold R, Jinek M. Structural basis of AAUAAA polyadenylation signal recognition by the human CPSF complex. eLife. 2018

15. Derti A, et al. A quantitative atlas of polyadenylation in five mammals. Genome Res. 2012; 22:1173-83. [PubMed: 22454233]

16. Gruber AJ, et al. A comprehensive analysis of $3^{\prime}$ end sequencing data sets reveals novel polyadenylation signals and the repressive role of heterogeneous ribonucleoprotein $\mathrm{C}$ on cleavage and polyadenylation. Genome Res. 2016; 26:1145-59. [PubMed: 27382025]

17. Hu J, Lutz CS, Wilusz J, Tian B. Bioinformatic identification of candidate cis-regulatory elements involved in human mRNA polyadenylation. RNA. 2005; 11:1485-93. [PubMed: 16131587]

18. Sheets MD, Ogg SC, Wickens MP. Point mutations in AAUAAA and the poly (A) addition site: effects on the accuracy and efficiency of cleavage and polyadenylation in vitro. Nucleic Acids Res. 1990; 18:5799-805. [PubMed: 2170946]

19. Guo Z, Sherman F. 3'-end-forming signals of yeast mRNA. Mol Cell Biol. 1995; 15:5983-90. [PubMed: 7565751]

20. Sun Y, et al. Molecular basis for the recognition of the human AAUAAA polyadenylation signal. Proc Natl Acad Sci U S A. 2017

21. Gradia SD, et al. MacroBac: New Technologies for Robust and Efficient Large-Scale Production of Recombinant Multiprotein Complexes. Methods Enzymol. 2017; 592:1-26. [PubMed: 28668116]

22. Biyani N, et al. Focus: The interface between data collection and data processing in cryo-EM. $\mathbf{J}$ Struct Biol. 2017; 198:124-133. [PubMed: 28344036]

23. Zheng SQ, et al. MotionCor2: anisotropic correction of beam-induced motion for improved cryoelectron microscopy. Nat Methods. 2017; 14:331-332. [PubMed: 28250466]

24. Rohou A, Grigorieff N. CTFFIND4: Fast and accurate defocus estimation from electron micrographs. J Struct Biol. 2015; 192:216-21. [PubMed: 26278980]

25. Scheres SH. RELION: implementation of a Bayesian approach to cryo-EM structure determination. J Struct Biol. 2012; 180:519-30. [PubMed: 23000701]

26. Kucukelbir A, Sigworth FJ, Tagare HD. Quantifying the local resolution of cryo-EM density maps. Nat Methods. 2014; 11:63-5. [PubMed: 24213166]

27. Emsley P, Cowtan K. Coot: model-building tools for molecular graphics. Acta Crystallogr D Biol Crystallogr. 2004; 60:2126-32. [PubMed: 15572765]

28. Afonine PV, Headd JJ, Terwilliger TC, Adams PD. New tool: phenix.real_space_refine. Computational Crystallography Newsletter. 2013; 4:43-44.

29. Pettersen EF, et al. UCSF Chimera--a visualization system for exploratory research and analysis. J Comput Chem. 2004; 25:1605-12. [PubMed: 15264254] 
a

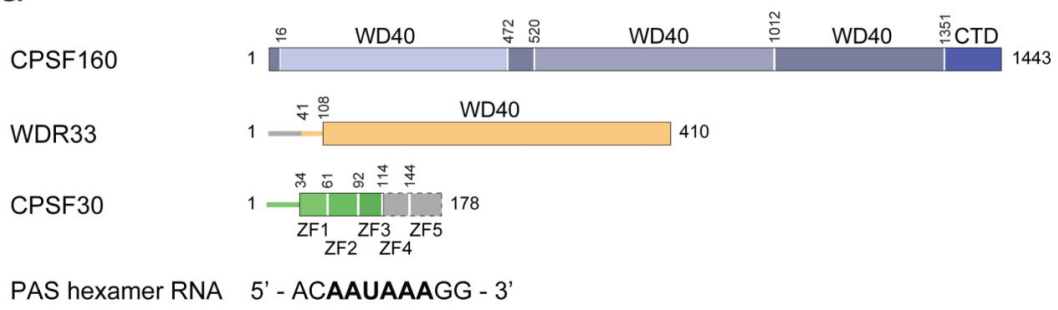

b

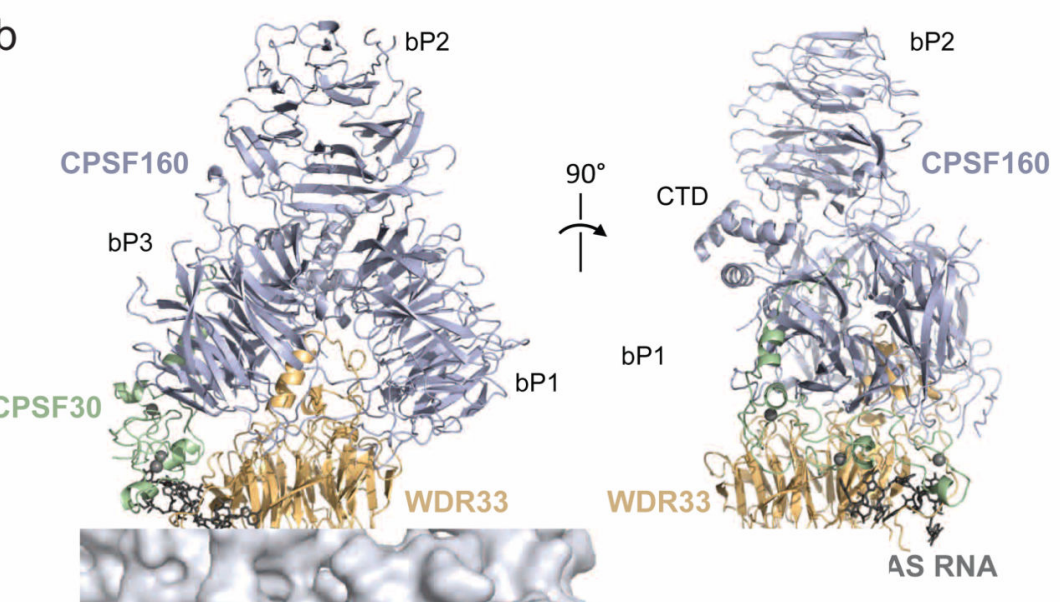

C

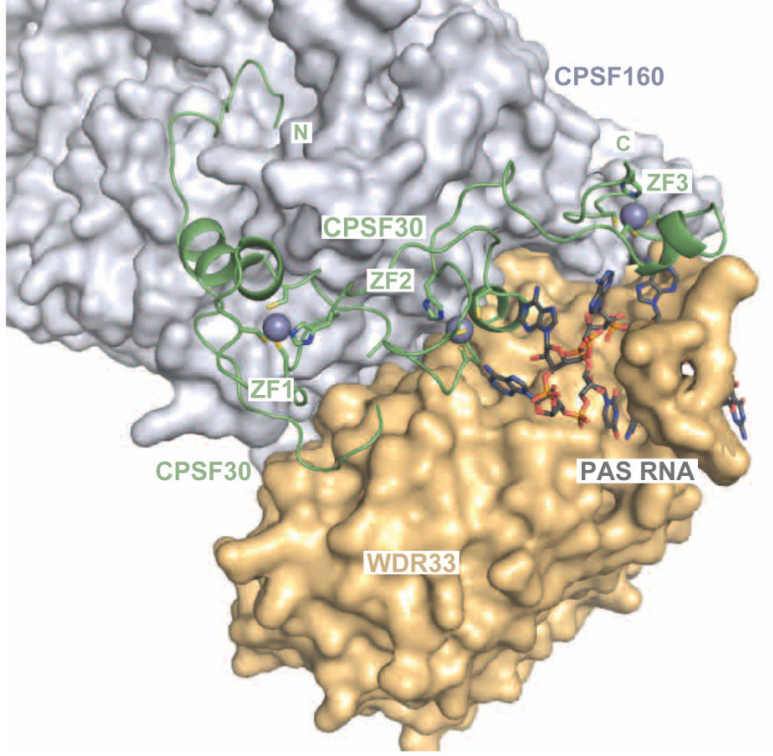

Figure 1. Molecular architecture of CPSF160-WDR33-CPSF30 in complex with the AAUAAA PAS hexamer motif.

(a) Schematic diagram depicting CPSF160, WDR33 and CPSF30 constructs used for complex reconstitution (not to scale). $\mathrm{N}$ - and $\mathrm{C}$-terminal regions for which no electron density was observed are depicted in grey. CPSF160 beta-propeller domains (bP1-3) and Cterminal domain (CTD) are highlighted with different shades of blue. CPSF160 regions forming beta-propeller domain 3 (bP3) are non-contiguous in the primary structure of the protein. (b) Front (left) and side (right) views of the CPSF160-WDR33-CPSF30-AAUAAA 
hexamer motif complex shown in cartoon (proteins) and stick (RNA) format. CPSF160 betapropeller domains and C-terminal domain are indicated as bP1 to bP3 and CTD, respectively. Zinc atoms are displayed as grey spheres. (c) Zoomed-in view of CPSF30 binding to a cleft formed by CPSF160 C-terminal beta-propeller (bP3) and WDR33. CPSF30 is represented as cartoon, PAS RNA as sticks and CPSF160-WDR33 as surface. Zinc atoms are displayed as grey spheres. 


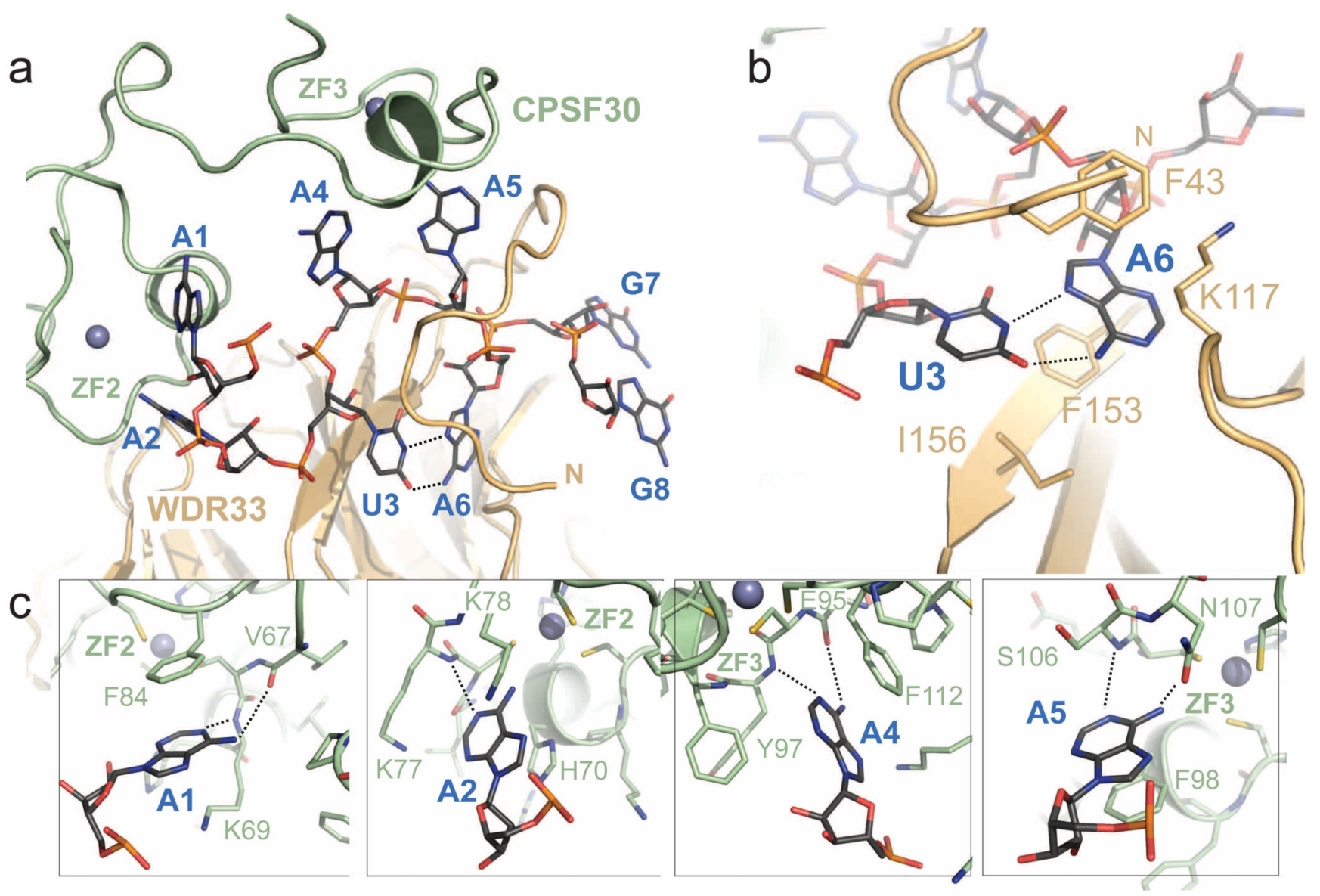

Figure 2. AAUAAA motif recognition by CPSF30 and WDR33.

(a) Overall view of PAS hexamer RNA recognition by CPSF30 ZNF2 and ZNF3 domains and WDR33. CPSF30 and WDR33 are shown in cartoon format the PAS RNA as sticks. Hoogsteen base-pair hydrogen bonds between nucleobases of U3 and A6 are indicated as dotted lines. (b) Zoomed-in view of the U3-A6 Hoogsteen base-pair sandwiched between WDR33 F43 and F153/I156 residues. Hydrogen bonds are indicated as dotted lines. (c) Recognition of nucleotides A1, A2, A4 and A5 by CPSF30 ZNF2 and ZNF3 domains. 